26. Glatzmaier, G. A. J. comput. Phys. 55, 461-484 (1984).

27. Glatzmaier, G. A. Astrophys. J. 291, 300-307 (1985).

28. Glatzmaier, G. A. Geophys astrophys. Fluid Dyn 31, 137-150 (1985).

29. Kageyama, A. et al. Phys. Plasmas 2, 1421-1431 (1995).

30. Glatzmaier, G. A. \& Roberts, P. H. Phys. Earth planet. Inter. 91, 63-76 (1995)

31. Rikitake, T. Proc. Camb. phil. Soc. 54, 89-105 (1966).

32. Bloxham, J. \& Gubbins, D. Nature 317, 777-781 (1985)

33. Cain, J. C., Wang, Z., Schmitz, D. R. \& Meyer, J. Geophys. J. 97, $443-447$ (1989).

34. Langel, R. in Geomagnetism (ed. Jacobs, J. A.) Vol. 1, 249-512 (Academic, San Diego, 1987).

35. Coe, R. S., Prevot, M. \& Camps, P. Nature 374, 687-692 (1995).

36. Hoffman, K. A. Nature 359, 789-794 (1992).

37. Opdyke, N. D., Kent, D. V. \& Lowrie, W. Earth planet. Sci. Lett. 20, 315-324 (1973).

38. Kristjansson, L. Geophys. J. R. astr. Soc. 80, 57-71 (1985).

39. Tric, E. et al. Phys. Earth planet. Inter. 65, 319-336 (1991)

40. Clement, B. M. Earth planet. Sci. Lett. 104, 48-58 (1991).
41. Laj, C., Mazaud, A., Weeks, R., Fuller, M. \& Herrero-Bervera, E. Nature 351, 447 (1991). 42. Ratcliff, C. D., Geissman, J. W., Perry, F. V., Crowe, B. M. \& Zeitler, P. K. Science 266, 412 416 (1994).

43. Langeres, C. G., van Hoof, A. A. M. \& Rochette, P. Nature 358, 226-230 (1992).

44. Valet, J.-P., Tuchloka, P., Courtillot, V. \& Meynadier, L. Nature 356, 400-407 (1992).

45. McFadden, P. L., Barton, C. E. \& Merrill, R. T. Nature 361, 342-344 (1993).

46. Prevot, M. \& Camps, P. Nature 366, 53-57 (1993).

47. McFadden, P. L. \& Merrill, R. T. J. geophys. Res. 100, 307-316 (1995)

48. Runcorn, S. K. Nature 356, 654-656 (1992).

49. Clement. B. M. \& Stixrude, L. Earth planet. Sci. Lett. 130, 75-85 (1995).

50. Valet, J.-P. \& Meynadier, L. Nature 366, 234-238 (1993).

51. Clement, B. M. \& Kent, D. V. Geophys. Res. Lett. 18, 81-84 (1991)

ACKNOWLEDGEMENTS. The computing resources for this simulation were provided by the NSF Pittsburgh Supercomputing Center. Support for this research was provided by the Institute of Geophysics and Planetary Physics and the LDRD program at Los Alamos.

\title{
Transcription complex stability and chromatin dynamics in vivo
}

\author{
Mark Wijgerde ${ }^{*}$, Frank Grosveld ${ }^{* \dagger} \&$ Peter Fraser \\ * MGC Department of Cell Biology and Genetics, Erasmus University, 3015 GE Rotterdam, The Netherlands \\ † Laboratory of Gene Structure and Expression, National Institute for Medical Research, The Ridgeway, Mill Hill, London NW7 1AA, UK
}

Distant regulatory sequences affect transcription through long-range chromatin interactions. Visualization of transcriptional activity of genes that compete for distant elements, using the globin locus as a model, has revealed the dynamics of chromatin interactions in vivo. Multiple genes appear to be transcribed alternately rather than at the same time to generate several messenger RNAs in one cell. The regulator may stably complex with one gene at a time and switch back and forth between genes in a flip-flop mechanism.

MANY genes are dependent for expression on the presence of distant regulatory elements which may be tens of thousands of base pairs away. The five human $\beta$-globin genes are arranged in the order of their developmental expression $(\varepsilon-G \gamma-A \gamma-\delta-\beta)^{1}$ and all are dependent on the locus control region (LCR) for highlevel, position-independent expression ${ }^{2-4}$. The LCR is located over 50 kilobases $(\mathrm{kb})$ upstream of the $\beta$-globin gene and consists of five DNase I-hypersensitive sites (HS) ${ }^{3,5} 7$. The most important sites in terms of transcriptional activation are HS 2-4 (refs 8, 9 and $\mathrm{J}$. Ellis et al., manuscript submitted), each having a core region of $200-300$ base pairs (bp $)^{10-14}$.

Transcriptional competition between genes ${ }^{15,16}$ is important in vivo in determining the pattern of globin gene expression during development ${ }^{1720}$ (N. Dillon et al., manuscript submitted). All of the genes are in polar competition for the activating function of the LCR at all stages of development, with proximal genes having an advantage over distal genes, suggesting that the LCR interacts directly with the gene(s) by a looping mechanism.

The distal $\beta$-globin gene is transcriptionally competent at all stages of development, but its expression is suppressed in embryonic erythroid cells by the LCR proximal $\varepsilon$ - and $\gamma$-globin genes. Expression of the $\beta$-gene occurs only after silencing of the $\varepsilon$ - and $\gamma$-genes, presumably by multiple silencing elements in the sequences immediately flanking the genes ${ }^{21-23}$. In contrast to models proposing that different HS regions of the LCR interact with different genes at the same time ${ }^{24}$, we have proposed that the LCR HS elements interact to act together or form a holocomplex which then interacts with proximal transcriptionally competent genes via looping (ref. 9 and J. Ellis et al., submitted). The LCR holocomplex model would explain why the activity of the HS is additive and why each of the genes requires all of the HS for full expression. Most important, it would explain the fact that there is balanced competition between the genes for LCR function. A key question in validating this model is whether the LCR is limited to activating only a single gene at a time or, as others suggest, the LCR splits its function ${ }^{24}$ and activates several genes simultaneously ${ }^{25,26}$. We have addressed this question by in situ hybridization analysis of the erythroid tissues derived from a transgenic mouse line carrying a single copy of the complete human $\beta$-globin locus. The results show that the LCR activates only one gene at a time, indicating that the LCR-globin-gene interaction is monogenic. Furthermore, these interactions are not static, but dynamic, with transcription interactions forming, breaking and reforming in a type of flipflop mechanism. Finally, the average stability of the LCR-gene interactions was estimated to be of the order of 15-80 min.

\section{Globin primary transcripts}

Analysis of $\gamma$ - and $\beta$-globin steady-state messenger RNA levels during switching in the early stages of transgenic fetal liver erythropoiesis (11.5-13.5 d) show that both are detectable in total fetal liver $\mathrm{RNA}^{27}$, with $\gamma$-gene expression decreasing and $\beta$-globin gene expression increasing. Both $\gamma$ - and $\beta$-globin polypeptides occur in most cells of the transgenic fetal liver ${ }^{9,28}$, indicating that commitment to $\gamma$ - or $\beta$-gene expression does not occur before the onset of globin transcription. The detection of globin mRNA or polypeptides are not reliable indicators of concurrent transcriptional activity in developing cells owing to the long half-lives of such molecules. Alternatively, primary transcripts are both temporally and spatially associated with the transcriptional event and have short half-lives due to rapid splicing into mature mRNA. The half-life of the mouse $\beta$-major globin primary transcript has been calculated to be $<5 \mathrm{~min}$ in mouse erythroleukaemia cells ${ }^{29}$. Detection of primary transcripts in situ is therefore an accurate indicator of ongoing or very recent transcription. If transcription complexes last longer than the half-life of the primary transcript, two types of signals may occur: single-gene transcription signals or multigene signals. Whereas the explanation for the single-gene signal is obvious, the multigene signal could result from either of two events, namely concurrent transcription from two genes or a recent switch causing a signal overlap between transcription from one 
FIG. 1 In situ hybridization of transgenic and nontransgenic mouse fetal liver cells. a-c, Hybridization of homozygous 12.5-day mouse fetal liver cells (line 72$)^{27}$ containing two single human $\beta$-globin loci; a shows the red signal only (Texas red, $\gamma$ ), $b$ the green signal only (FITC, $\beta$ ), and $c$ the overlay of both after laser confocal microscopy. $d, e$, same as $c$, but in $d$ cells have first been treated with RNase; e shows results from a non-transgenic fetal liver. $f$, Quanitification of the different types of transgenic mouse 12.5-day fetal liver cells. Red (Texas red) represents the $\gamma$ signal, green (FITC) the $\beta$ signal, and yellow the combination of both. Scoring was by epifluorescence on a minimum of 400 cells per liver sample and at least two livers. The frequency of occurrence of each cell type is shown as a percentage.

METHODS. 12.5-day fetal livers were disrupted into PBS and the cells fixed onto a poly-L-lysine coated slide in $4 \%$ formaldehyde $/ 5 \%$ acetic acid for $20 \mathrm{~min}$ at room temp. ${ }^{35}$. Cells were subsequently washed 3 times for $10 \mathrm{~min}$ in PBS and stored in $70 \%$ ethanol at $-20{ }^{\circ} \mathrm{C}$. Slides were pretreated for hybridization by a $0.01 \%$ pepsin digestion $\left(5 \mathrm{~min}, 37^{\circ} \mathrm{C}\right.$ ) in $0.01 \mathrm{M} \mathrm{HCl}$, followed by a short wash in water and a 5-min fixation in 3.7\% formaldehyde at room temperature, then washed in PBS, dehydrated in 70,90 and $100 \%$ ethanol steps and air-dried. The hybridization mixture was applied (12 $\mu$ l per $24 \times 24 \mathrm{~mm}$ coverslip) and incubated at $37^{\circ} \mathrm{C}$ in a moisturized chamber for $12 \mathrm{~h}$.
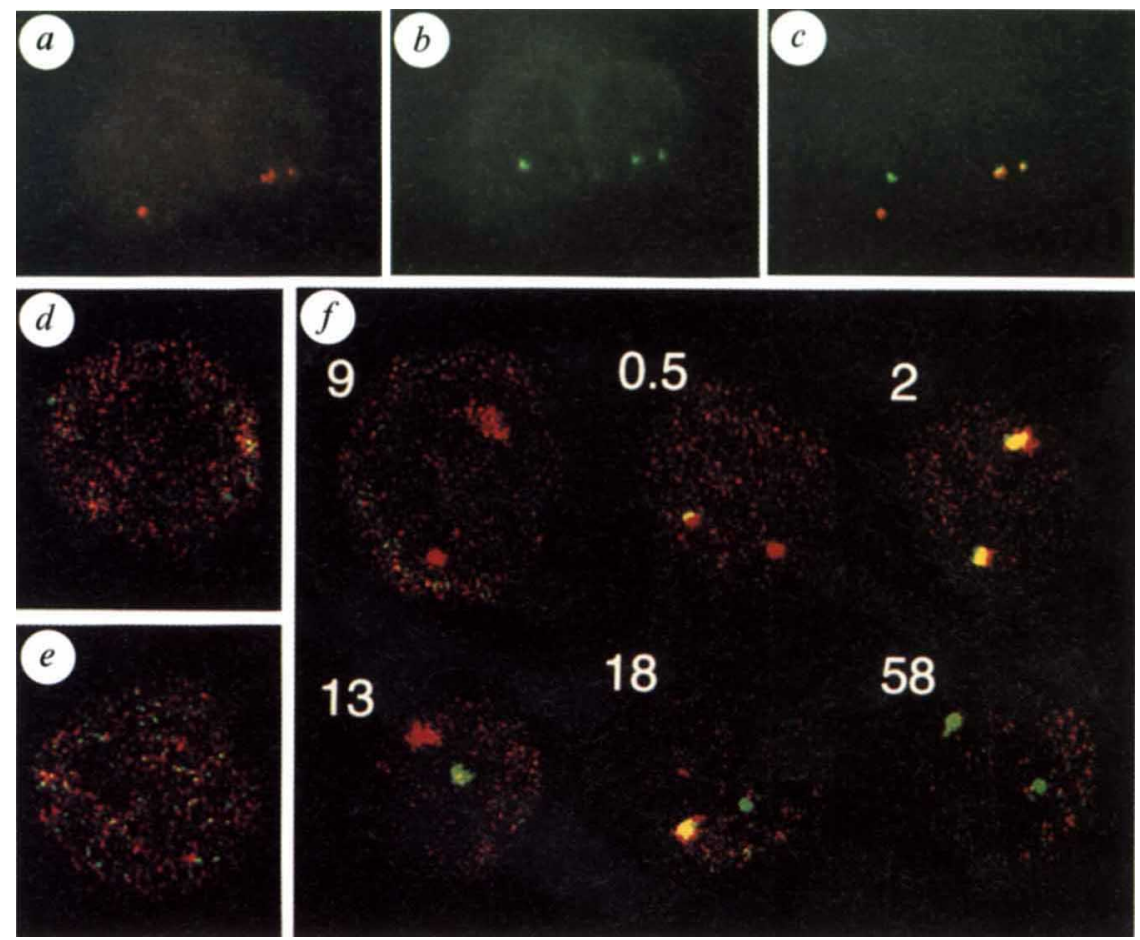

each of three or four oligonucleotides (50 nucleotides long) containing a DNP, a digoxygenin or a biotin side chain in the middle and on the $5^{\prime}$ and the $3^{\prime}$ end of the oligonucleotide (Eurogentec, Belgium) in 25\% formamide, $2 \times$ SSC, salmon sperm DNA (200 ng $\left.\mu^{-1}\right), 5 \times$ Denhardts, $1 \mathrm{mM}$ EDTA and $50 \mathrm{mM}$ sodium phospate, $\mathrm{pH}$ 7.0. The oligonucleotide sequences were derived from the first and second introns of the globin genes and spaced more than 25 nucleotides apart. The coverslip was removed by dipping in $2 \times$ SSC and the cells were washed three times

gene and the decay of the pre-mRNA (processing) from the other gene.

We have used in situ hybridization with gene-specific intron probes to localize human globin primary transcripts in nuclei of individual embryonic and fetal transgenic erythroid cells. The signals appear in the nucleus as fluorescent foci at the location of an actively transcribing gene $\mathrm{e}^{30,31}$. The foci do not appear in RNase-pretreated cells or in non-globin-expressing transgenic cells or non-transgenic cells (Fig. $1 d, e$ ), indicating that the probes specifically detect an intron-containing RNA molecule (the primary transcript). Probe penetration is almost complete as $>97 \%$ of the active mouse $\alpha$-globin genes can be detected (not shown). Heterozygous cells show single foci, whereas homozygous cells show two foci, demonstrating detection of each locus (not shown). To determine whether the LCR can activate the $\gamma$ - and $\beta$-globin genes simultaneously, we performed in situ hybridizations with gene-specific intron probes for $\gamma$ - and $\beta$ globin primary transcripts in homozygous day-12 transgenic mouse fetal liver cells (Fig. $1 a-c$ ). The cell on the right contains primary transcript signals for both $\gamma-$ (red) and $\beta$-globin (green) from each chromosome, suggesting that the LCR can activate both genes simultaneously from a single chromosome, as would be expected for co-transcription. Alternatively, the double signal could be due to an overlap between transcription of one gene and the decay of the pre-mRNA signal from the other gene, as would be expected for single-gene transcription. However, only the latter explanation would also fit the type of cell on the left, which is transcribing only $\gamma$-globin from one chromosome and only $\beta$-globin from the other. This suggests that the LCR activation mechanism is mono-gene-specific. Considering that these nuclei contain the transacting factors required for both $\gamma$ - and $\beta$-globin transcription, co-transcription would be unlikely if the in $2 \times \mathrm{SSC}$ at $37^{\circ} \mathrm{C}$, followed by a 5 -min wash in $0.1 \mathrm{M}$ Tris, $0.15 \mathrm{M}$ $\mathrm{NaCl}, 0.05 \%$ Tween 20 . Antibody detection of the labels was essentially as described by Dirks et al. ${ }^{35}$, with three or four amplification steps. Mounting was in DAPI/DABCO:Vectashield (1:1) in glycerol $(90 \%)$ epifluorescence/CCD or laser confocal microscopy. RNase treatment was in $0.1 \mathrm{M}$ Tris, $0.15 \mathrm{M} \mathrm{NaCl}, 10 \mu \mathrm{g} \mathrm{ml}{ }^{-1}$ RNase A for $5 \mathrm{~min}$ at room temperature.

$\gamma$ and $\beta$-only cell (left) were to occur frequently, given the high probe penetration $(>97 \%)$. We therefore quantified the different cell types.

\section{A dynamic mechanism}

Day-12 homozygous transgenic fetal liver cells show all possible primary transcript signal combinations of $\gamma$ - and $\beta$-globin (Fig. $1 f): 58 \%$ of globin-transcribing nuclei have only $\beta$-globin transcription on both chromosomes and $9 \%$ have only $\gamma$-globin; the remaining one third of the erythroid cells contain combinations of $\gamma$ - and $\beta$-globin transcription and are therefore involved in the switching process. However, fewer than half $(34 \%)$ of the chromosomes in these switching cells have both $\gamma$ - and $\beta$-globin signals on the same chromosome. The majority have single gene signals only, suggesting that the LCR interaction is largely mono-gene-specific. Of particular interest is the fact that in $>90 \%$ of the switching cells, the two chromosomes are responding differently to the same transacting-factor environment. This suggests a dynamic, continuously changing system in which the individual loci respond stochastically to changes in the factor environment.

\section{Flip-flop}

Two forms of such a dynamic process can be envisaged. Either the switch in interaction is progressive from $\gamma$ to $\beta$, or it switches back and forth between the genes in a kind of flip-flop mechanism. $\gamma$ - and $\beta$-globin signals on the same chromosome would then be indicative of a recent switch producing a (brief) period of overlap in which the decaying 'old' primary transcripts are detected in the presence of the newly synthesized transcripts. The length of this overlap period would depend on the half-life of the primary transcript. The progressive switching mechanism and stored at $4{ }^{\circ} \mathrm{C}$ in the dark. Fluorescence was detected by 

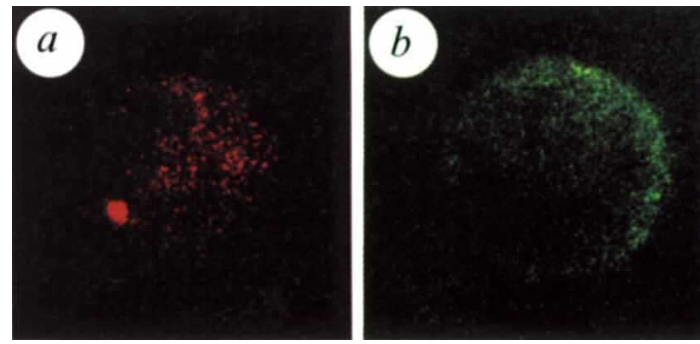

FIG. 2 Detection of $\gamma$-globin (Texas red) and $\beta$-globin (FITC, green) precursor mRNA in the nucleus and $\beta$-globin mRNA in the cytoplasm (Cy3, yellow). The fetal liver was obtained from a transgenic 12.5-day embryo heterozygous for the human $\beta$-globin locus. $\beta$-globin mRNA was visualized by indirect immunofluorescence with Cy3 of DNP-labelled oligonucleotides. Cy3 emission is also red but at a different wavelength from Texas red and was therefore recorded separately and assigned

would predict that heterozygous $\gamma$-gene-transcribing cells should not have $\beta$-mRNA in their cytoplasm, whereas the flip-flop mechanism would result in a proportion of $\gamma$ (but not $\beta$ ) transcribing nuclei, which contain $\beta$ mRNA in their cytoplasm.

We therefore added a third probe which specifically recognizes the human $\beta$-globin mRNA (exon probe). Heterozygous 12.5 day transgenic fetal liver cells were used, needing only a single chromosome to switch. Figure 2 shows one of many heterozygous cells probed for the $\gamma$ - and $\beta$-globin primary transcripts and cytoplasmic $\beta$-globin mRNA. The $\gamma$ transcription signal is very strong (red in Fig. $2 a$ ) whereas the $\beta$-gene signal (green in Fig. $2 b$ ) is absent, indicating that the $\gamma$ gene is currently being transcribed, but the $\beta$ gene is not. The cytoplasm, however, shows an accumulation of $\beta$ mRNA, indicating previous transcriptional activity of the $\beta$-gene in that cell (Fig. $2 c$ ). In fact, $40 \%$ of the erythroid cells that are transcribing the $\gamma$ gene, but not the $\beta$ gene, contain $\beta$ mRNA. In other words, there is a substantial number of cells that have transcribed $\beta$ but switched back to $\gamma$, an observation that strongly supports a dynamic flip-flop between the genes. Many of the chromosomes with both $\gamma$ - and $\beta$-globin primary transcript signals (Fig. 2) would therefore be the result of flip-flop rather than co-transcription. Thus, the decay time of our signal after a switch becomes important in determining whether most or all of the overlap we observe is due to flip-flop. The $15 \mathrm{~S}$ mouse $\beta$-major globin primary transcript was shown to reach its steady-state level in MEL cells within $5 \mathrm{~min}^{29}$. This indicates that the half-life of the completely intact precursor RNA is considerably shorter as it takes $\sim 5$ half-lives to reach $97.5 \%$ of steady-state level. However, the target of our in situ experiments is not only the intact $15 \mathrm{~S}$ globin primary transcript, but also any other intron-containing RNA molecules such as partially transcribed, partially spliced and excised intron RNAs. We therefore measured the half-life of the intron-containing RNA molecules for mouse $\beta$-major globin and human $\gamma$ - and $\beta$-globin in day-12 transgenic fetal liver cells. The results indicate that all three have similar kinetics, reaching steady-state levels simultaneously (Fig. 3). The halflife of the intron is $4-5 \mathrm{~min}$, in agreement with Curtis et al. ${ }^{29}$. Assuming that we can still detect the in situ signal after 2-3 half-lives (see discussion), it suggests that the overlap period is $10-15 \mathrm{~min}$.

\section{Replication and switching}

One possibility to explain the occurrence of multigene signals when only a single gene is active at any time could be that it is the result of replication of a locus. Homozygous replicating cells (in late $\mathrm{S}, \mathrm{G} 2$ and $\mathrm{M}$ ) contain four globin loci in the nucleus and hence a $\gamma \beta$ co-transcription signal could be the result of the $\gamma$ gene being transcribed from one of the replicated loci, whereas $\beta$ could be transcribed from its replicated chromatid. This would result in the percentage of $\gamma \beta$ signals being substantially higher
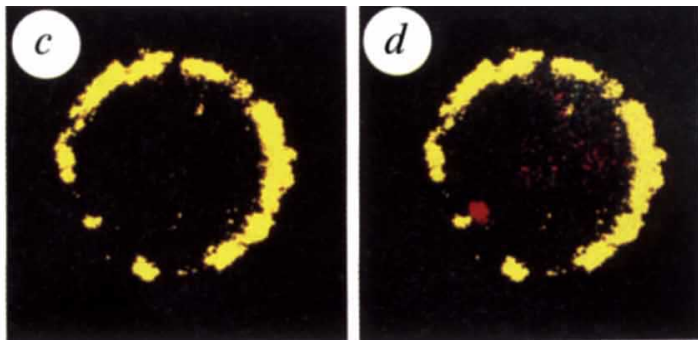

an artificial yellow. $a$, Texas red signal ( $\gamma$ pre-mRNA); $b$, FITC green signal ( $\beta$ pre-mRNA) - this image was digitally enhanced to demonstate the lack of a $\beta$ transcript, so some bleed-through from the cytoplasmic Cy3 signal appears as a high background; $c$, Cy 3 signal ( $\beta$ mRNA). This is a single optical section of the cell from the confocal microscope $(0.5$ microns); $d$ is a composite of $a, b$ and $c$.

in G2 than in G1 cells. We therefore sorted 12.5-day fetal liver into populations of G1 and G2 cells ${ }^{32}$ and repeated the hybridizations. Analysis of the G1 and G2 cells shows a distribution and percentage of $\gamma \beta$ signals similar to the total population, with no significant difference between the two purified populations. We conclude that the switch between genes can take place in G1-that is, DNA replication is not required, implying that the LCR-gene interactions are dynamic rather than static. As expected $^{33,34}$, no transcription signals are observed when the cells are in mitosis.

\section{Co-expression and switching}

To extend these results, we characterized the transcriptional status of individual cells at different times of development,

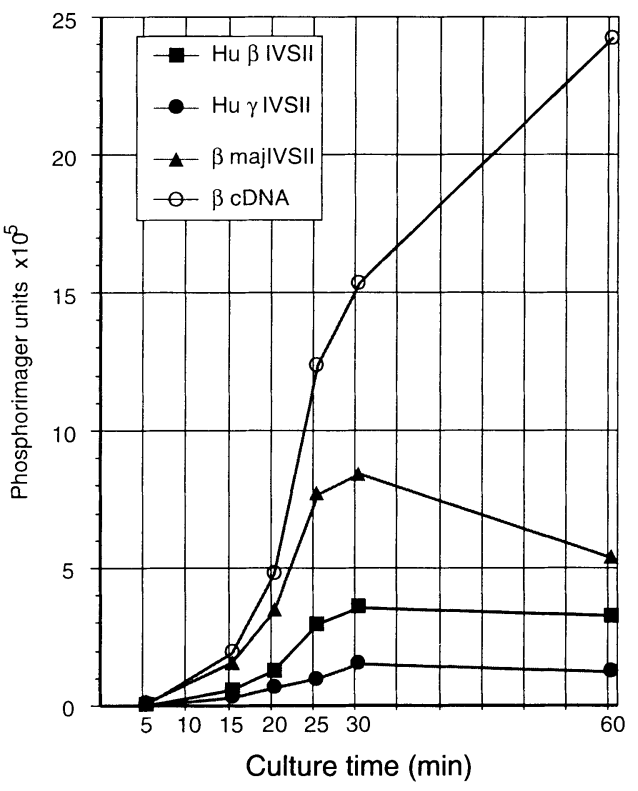

FIG. 3 Incorporation of ${ }^{32} \mathrm{P}$ into globin intron and mRNA sequences. 12.5-day fetal liver cells from heterozygous line 72 were cultured in the presence of ${ }^{32} \mathrm{P}$ for the times indicated, essentially as described ${ }^{29}$. Halflives were calculated by comparing the difference in the rates of incorporation between intron and exon sequences. The 4-5-min halflife fits well with the fact that 5 half-lives are required to reach $97.5 \%$ of the steady state level.

METHODS. RNA was prepared for $1.5 \times 10^{7}$ cells per time point and hybridized to $5 \mu \mathrm{g}$ immobilized denatured plasmid DNA as described ${ }^{36}$. Results were quantified by phosphorimage analysis. Plasmids contained mouse $\beta$-major intron II (BamHI/Pstl), human $\beta$ intron II (BamHI/Sspl), human $\gamma$ intron II (BamHI/Sacl) and partial human $\beta$-globin cDNA; signals were normalized for relative probe length. 


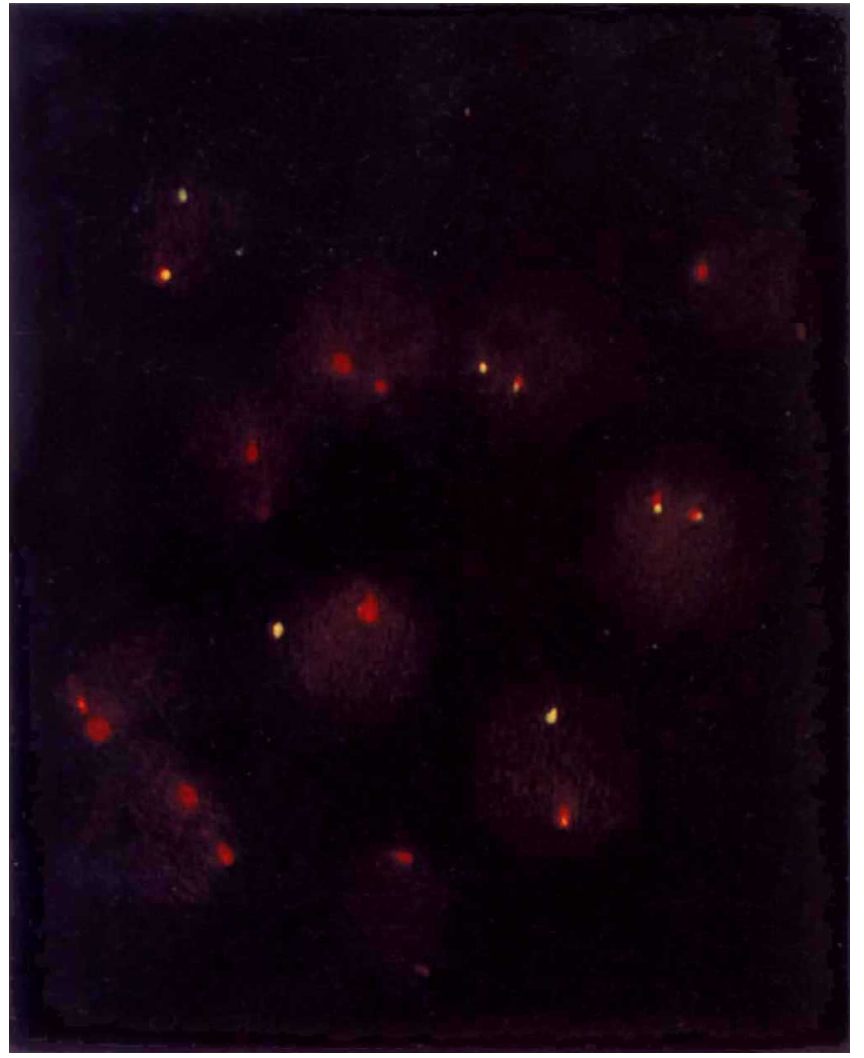

FIG. 4 Simultaneous detection of $\varepsilon$ (FITC, green) and $\gamma$ (Texas red) transcription in 10-day embryonic blood. Cells were obtained from a line-72 embryo homozygous for the human globin locus ${ }^{27}$.

namely day-10 embryonic blood and day-11 fetal liver. During transgenic embryonic erythropoiesis $\gamma$ - and $\varepsilon$-globin expression occurs simultaneously over a period of at least 3 days (days 8 10 of gestation) and nearly all cells contain both $\varepsilon$ - and $\gamma$-globin polypeptides $^{28}$. We have previously determined the level of expression of the $\gamma$-and $\varepsilon$-globin genes when present as single genes linked to the LCR and compared this to the level of expression when both are linked together to the LCR. That comparison suggested that the $\gamma$ and $\varepsilon$ genes are also in competition for the LCR (P.F., unpublished results), presumably through a looping mechanism (N. Dillon et al., submitted). The circulating embryonic erythrocytes are nucleated and transcriptionally active, and hence were used to determine whether flip-flop could also be occurring between the $\varepsilon$ - and $\gamma$-globin genes by probing day-10 embryonic erythrocytes with gene-specific intron probes for $\varepsilon$ and $\gamma$ (Fig. 4). The results demonstrate (like $\gamma$ and $\beta$ in the fetal liver) a heterogeneity in transcriptional signals, including chromosomes with overlapped signals (Figs 4 and 5). We conclude that the LCR also productively interacts with only one gene at a time in peripheral embryonic blood cells and that $\varepsilon$ and $\gamma$ are co-expressed via a flip-flop mechanism. The higher incidence of overlapped signals on individual chromosomes suggests that the LCR $-\varepsilon$ complex is less stable than those of $\gamma$ and $\beta$ with the LCR (see discussion).

The analysis of day-11 fetal liver shows that the switching process is a continuously changing system, reflected at the level of the individual cell (Fig. 5). When compared to the day-12 results, there are more $\gamma \gamma$ - and fewer $\beta \beta$-transcribing cells $(27$ versus $9 \%$ and 35 versus $58 \%$ ); accordingly, significantly more cells are observed that transcribe $\gamma$ only from locus and $\gamma$ and $\beta$ from the other locus ( 7 versus $<1 \%$ ). The number of cells transcribing $\gamma$ only on one chromosome and $\beta$ only on the other chromosome is very similar (14 versus $13 \%)$. These results indicate that the change in the composition of transcription factors during the switch is gradual and slowly changes the affinity between the LCR and the genes towards the adult type LCR/ $\beta$-globin gene interaction.

\section{Discussion}

We have demonstrated the dynamic nature of the interactions between the human $\beta$-globin genes and the $\beta$-globin LCR throughout development and shown that the LCR-gene interactions are highly stable. Our data can all be explained by a mechanism in which the LCR activates only a single gene in the locus at any given time. The LCR, although made up of a series of hypersensitive sites, could act as a functional unit or holocomplex that activates multiple genes from the same chromosome via a stochastic mechanism of dynamic rather than static interactions (Fig. 6). Although a low level of co-transcription cannot be excluded such a mechanism cannot account for our observations. Dillon et al. (manuscript submitted) have used steadystate measurements of the transcriptional output from mutated loci to demonstrate that the genes compete in a polar manner for the activating function of the LCR. They find that the relative distance of the genes from the LCR affects competition and conclude that the genes are activated by direct interaction with the LCR. Thus two studies, which use entirely different approaches, provide strong evidence of direct interaction between the LCR and single genes by a dynamic looping mechanism of transcriptional activation. It does not show whether some process spreading in a linear fashion along the chromatin is involved in the first activation of the locus. The balance of, $\gamma$-versus $\beta$-gene transcription would gradually change during a
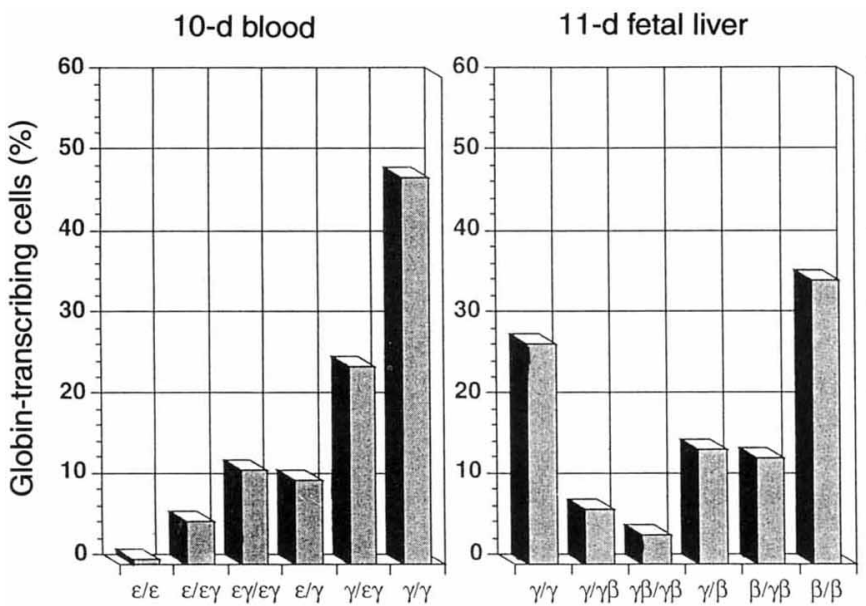

12-d fetal liver

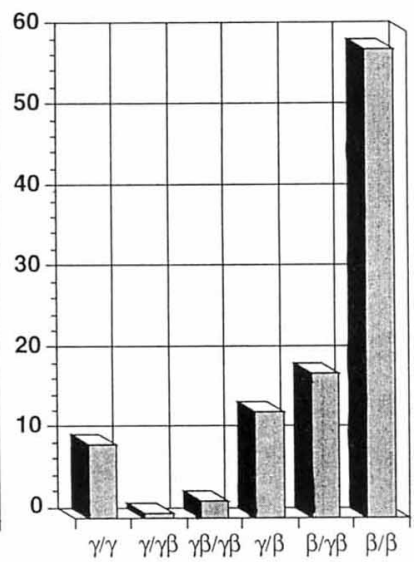

FIG. 5 Bar diagram of the percentages of transcriptional cell types observed at different times of development in transgenic cells homozygous for the human globin locus. A single transcription signal at one of the loci is indicated with a single letter, double signals with two letters. For example $\gamma / \gamma \beta$ is a cell type with a $\gamma$ signal only on one chromosome and signals for $\gamma$ and $\beta$ on the other chromosome. The number of cell types in the population are given as a percentage. 
FIG. 6 A model of the interactions of the locus control region (LCR) with the different globin genes through a stochastic looping mechanism. The LCR is indicated as a squiggly line to indicate that the different regions that are hypersensitive to DNase I of the LCR could act together or even form a holocomplex to establish an interaction of the LCR with one of the genes.

switching period as the factor composition changes in the population.

\section{Flip-flop time and complex stability}

By scoring the different transcriptional chromosome types in the erythroid nuclei (Fig. 5), it is possible to estimate the flip-flop time, or the average time required for the LCR-gene interaction to change from $\gamma$ to $\beta$ (or vice versa), based on the half-life of the intron-containing RNAs and the percentages of different transcriptional states. The half-life determines the decay rate of the signal and thus the overlap time, that is, the length of time both $\gamma$ - and $\beta$-signals are detectable on the same chromosome after a change in LCR-gene interaction. As the cell population is random (so the chromosomes are not synchronized in terms of the $\gamma$-to- $\beta$ switch), the percentage of chromosomes showing a double signal at any particular instant indicates the fraction of the total time in which a chromosome is in the overlapped state. By extrapolation, we can determine the average length of time that a gene is transcribed-that is, the time between flipflop events. For example, the frequency of overlapped $\beta$ signals in the population at 12 days is $13 \%$ of the total number of $\beta$ signals. Therefore, on average, $13 \%$ of the time in which an individual chromosome transcribes the $\beta$ gene it will be overlapped by a $\gamma$ signal. The overlap time can be estimated as 10 $15 \mathrm{~min}$ on the basis of the intron half-life of 4-5 min and the fact that we can barely detect the precursor RNA when we use one (instead of three or four) oligonucleotides (M.W., unpublished results). As $13 \%$ of the $\beta$ signal is overlap, the total $\beta$ gene transcription time would be $\sim 80 \mathrm{~min}$. (As overlap occurs at the beginning and end of a transcription period, the time in overlap

Received 20 February; accepted 4 August 1995.

1. Collins, F. S. \& Weissman, S. M. Prog. nucl. Acid Res. molec. Biol. 31, 315-462 (1984).

2. Kioussis, D. et al. Nature 306, 662-666 (1983).

3. Grosveld, F., Blom van Assendelft, G., Greaves, D. R. \& Kollias, G. Cell 51, 975-985 (1987).

4. Driscoll, M. C., Dobkin, C. S. \& Alter, B. P. Proc. natn. Acad. Sci. U.S.A. 86, 7470-7474 (1989).

5. Tuan, D., Solomon, W., Li, Q. \& London, I. M. Proc. natn. Acad. Sci. U.S.A. 82, 6384-6388 (1985).

6. Forrester, W. C., Novak, U., Gelinas, R. \& Groudine, M. Proc. natn. Acad. Sci. U.S.A. 86, 5439-5443 (1989)

7. Ryan, T. M. et al. Genes Dev. 3, 314-323 (1989)

8. Fraser. P. Hurst J Collis, P. \& Grosveld, F. Nucleic Acids Res. 18, 3503-3508 (1990)

9. Fraser, P., Pruzina, S., Antoniou, M. \& Grosveld, F. Genes Dev. 7, 106-113 (1993).

10. Philipsen, S., Talbot, D., Fraser, P. \& Grosveld, F. EMBO J. 9, 2159-2167 (1990)

11. Talbot, D., Philipsen, S., Fraser, P. \& Grosveld, F. EMBO J. 9, 2169-2178 (1990).

12. Ney, P. A., Sorrentino, B. P., McDonagh, K. T. \& Nienhuis, A. W. Genes Dev. 4, 993-1006 (1990).

13. Pruzina, S. et al. Nucleic Acids Res. 19, 1413-1419 (1991)

14. Strauss, E. \& Orkin, S. Proc. natn. Acad. Sci. U.S.A. 89, 5809-5813 (1992)

15. de Villiers, J., Olson, C., Banerji, J. \& Schaffner, W. Cold Spring Harbor Symp. quant. Biol. 47, 911-919 (1982).

16. Wasylyk, B., Wasylyk, C., Augereau, P. \& Chambon, P. Cell 32, 503-514 (1983).

17. Choi, O. \& Engel, J. Cell 55, 17-26 (1988).

18. Behringer, R. R. et al. Genes Dev. 4, 380-389 (1990).

19. Enver, T. et al. Nature 344, 309-313 (1990).

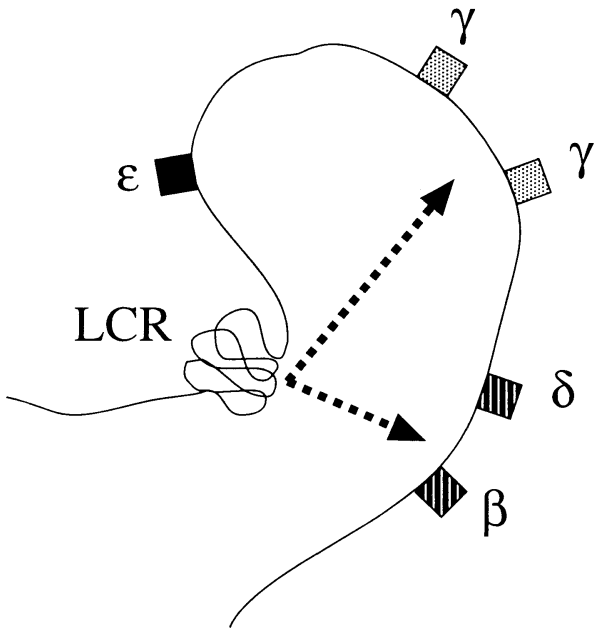

is $2 \times 10-15 \mathrm{~min}$. The total transcription time is therefore $100 /$ $13 \times 12$ min minus one overlap of $12 \mathrm{~min}$.) Correspondingly, the percentage of overlapped $\gamma$ signals is $42 \%$, making the average $\mathrm{LCR} / \gamma$ gene time between flip-flops $\sim 16 \mathrm{~min}$. When the results are compared for $\varepsilon, \gamma$ and $\beta$ from days $10-12$, several trends become apparent. First, at 10 days, the $\varepsilon$ signal is overlapped with a $\gamma$ signal $75 \%$ of the time, which suggests that the lifetime of the $\varepsilon$-LCR complex is short (about $4 \mathrm{~min}$ ) and flip-flop occurs more readily. The $\gamma$ signal is present more often than $\varepsilon$ at 10 days and is overlapped only $29 \%$ of the time, suggesting that the $\gamma$-LCR complex is more stable, with $\sim 30$ min between flipflops. The average stability of the $\gamma$-LCR complex decreases from 10 to 12 days from 30 to 16 min, whereas $\beta$ increases from 45 to $80 \mathrm{~min}$ from 11 to 12 days. Clearly the time between flipflops decreases for $\gamma$ but increases for $\beta$. Obviously interaction could consist of rapid associations and dissociations of the LCR with a given gene, rather than one stable association. Whatever the mechanism, our data suggest that the continuous presence of the regulatory elements at a gene are required for multiple rounds of initiation of transcription of that gene, rather than being dispensable after the activation of transcription.

In summary, our technique has enabled us to analyse single genes in single cells and allows us to make the conclusion that $\gamma$ - and $\beta$-globin genes are transcribed alternately rather than concurrently. The ability to visualize specifically the transcriptional activity of individual genes in the nucleus provides a powerful tool for studying developmental transcriptional regulation and chromatin dynamics and should provide a more accurate picture of transcriptional regulation compared with conventional in situ analysis.

20. Hanscombe, O. et al. Genes Dev. 5, 1387-1394 (1991)

21. Dillon, N. \& Grosveld, F. Nature 350, 252-254 (1990).

22. Lindenbaum, M. \& Grosveld, F. Genes Dev. 4, 2075-2085 (1990).

23. Raich, N. et al. Science 250, 1147-1149 (1990)

24. Engel, D. Trends genet. Sci. 9, 304-309 (1993).

25. Bresnick, E. H. \& Felsenfeld, G. Proc. natn. Acad. Sci. U.S.A. 91, 1314-1317 (1994).

26. Furukawa, T., Zitnik, G., Leppig, K., Papayannopoulou, T. \& Stamatoyannopoulos, G. Blood 83, 1412-1419 (1994).

27. Strouboulis, J., Dillon, N. \& Grosveld, F. Genes Dev. 6, 1857-1864 (1992).

28. Strouboulis, J. thesis, Open Univ., UK (1994).

29. Curtis, P., Mantei, N. \& Weissmann, C. Cold Spring Harbor Symp. quant. Biol. 42, 971 984 (1977).

30. Lawrence, J. B., Singer, R. H. \& Marselle, L. M. Cell 57, 493-502 (1989).

31. Zhang, G., Taneja, K. L., Singer, R. H. \& Green, M. R. Nature 372, 22-29 (1994).

32. Arndt-Jovin, D. \& Jovin, T. J. hist. Cytochem. 25, 585-589 (1985).

33. Prescott, D. \& Bender, M. Expl Cell Res. 26, 260-268 (1962).

34. Terasima, T. \& Tolmach, L. Expl Cell Res. 30, 344-362 (1963)

35. Dirks, R., van de Rijke, F., Fujishita, S., van der Ploeg, M. \& Raap, A. J. Cell Sci. 104, 1187 1197 (1993).

36. Fraser, P. \& Curtis, P. J. Genes Dev. 1, 885-861 (1987).

ACKNOWLEDGEMENTS. We thank M. Antoniou for his help at the start of this project and with the design of the probes; $K$. Weiss, G. Paolella and A. Lamond for assistance with the EMBL confocal microscope; N. Dillon for suggestions; T. Verkerk for cell sorting; L. Braam for animal care; T. Trimborn for assistance; and Eurogentec (Belgium) for help with the probes. M.W. is supported by the NWO (The Netherlands). This work was supported by the NWO (NL), the MRC (UK) and the Howard Hughes Foundation (USA). 